\title{
Zero-lag white noise vector bilinear autoregressive time series models
}

\author{
E H Etuk and I A Iwok \\ Department of Mathematics and Computer Science, \\ Rivers State University of Science and Technology, \\ Port Harcourt', Nigeria
}

\begin{abstract}
The non linear part of a mixed bilinear time series structure seems to pose difficulty if we are to extract the pure autoregressive (AR) bilinear form from the mixed process with the condition that the outcome of such extraction clearly defines itself as an extension from its parent linear AR model. It is therefore of immense interest to address a ' bilinear' situation where the same "order" identified in the linear AR processes are extended to cover the linear and non linear components of a bilinear process with an exception that the lagged white noise process is allowed to remain in its present state. This research focused on these two innovations where the white noise is lagged zero to isolate a pure vector AR bilinear model from a mixed process based on the distribution of autocorrelation and partial autocorrelation function of the different series involved in a vector process, and the extension of the linear 'orders' to bilinear 'orders'. To achieve the aforementioned, we formulated a matrix for a general case of n-dimensional vector for an AR process and then considered a special case of zero lag of white noise. With given conditions, and introduction of diagonal matrix of lagged vector elements, special bilinear expressions reflecting the same 'orders' of the corresponding linear forms emerged. The zero lagged white noise denoted by $\varepsilon_{i t-0}$ clearly defined our models as pure AR bilinear models since the lag $I=0$ and is equivalent to the current state white noise $\varepsilon_{t}$ of the linear AR process. These gave a brilliant meaning to vector bilinear AR processes in terms of linear AR 'orders'. The workability of these special bilinear models was assessed by applying them to revenue series and the result showed that the models gave a good fit, in support of our idea.
\end{abstract}

Keywords: Autoregressive time series, Moving average process, Autoregressive-moving average process, Vector time series, Bilinear Autoregressive moving average time series model, Vector Bilinear process.

\section{INTRODUCTION}

Historically, the modeling of time series started with its linear forms and could be traced back to such models as Yule's (1927) autoregressive (AR) models. Since then such models have found wider applications and theoretical development. In many practical situations, however, the classical linear models are not appropriate for modeling most nonstationary series. This calls for the emergence of non linear models of which bilinear forms a class.

Maravall (1983) used a bilinear model to forecast Spanish monetary data and reported a near 10\% improvement in one-step ahead mean square forecast errors over several autoregressive moving average (ARMA) alternatives. There is no gain saying the fact that most of the economic or financial data assume fluctuations due to certain factors. That is why the use of non linear models in forecast gives higher precision than linear models.

A bilinear process according to Granger and Anderson (1978) takes the form:

$X_{t}=\sum_{i=1}^{p} a_{i} X_{t-i}+\sum_{j=1}^{q} c_{j} X_{t-j}+\sum_{k=1}^{P} \sum_{j=1}^{Q} b_{k j} X_{t-k} \varepsilon_{t-j}+\varepsilon_{t}$

Rao (1980); describes bilinear time series model $B L(p, r, m, k)$ as given by the difference equation:

$$
X(t)+\sum_{j=1}^{p} a_{j} X(t-j)=\sum_{j=1}^{r} c_{j} e(t-j)+\sum_{l=1}^{m} \sum_{l^{\prime}=1}^{k} b_{l^{\prime}} X(t-l) e\left(t-l^{1}\right)
$$

where $\{e(t)\}$ is an independent white noise process and $c_{0}=1 .\{X(t)\}$ is termed the bilinear process. The autoregressive moving average model 
$\operatorname{ARMA}(p, r)$ is obtained from (2) by setting $b_{l l^{1}}=0 \quad \forall l$ and $l^{1}$.

Parameter estimation of bilinear processes has been studied for particular cases by Bouzaachane et al. (2006). Boonchai and Eivind

(2005) gave the general form of multivariate bilinear time series models as :

$X(t)=\sum A_{i} \cdot X(t-i)+\sum M_{j} \cdot e(t-j)+\sum \sum \sum B_{d i j} X(t-i) \cdot e_{d}(t-j)+e(t)$

Here the state $X(t)$ and noise $e(t)$ are n-vectors and the coefficients $A_{i}, M_{j}$ and $B_{d i j}$ are $\mathrm{n} \times \mathrm{n}$ matrices. If all $B_{d i j}=0$, we have the class of wellknown vector $A R M A$-models.

In the various bilinear models above, the 'orders' of the linear parts are different from those of the non linear parts. For example in equation (2) above, the orders of the linear parts are $r$ and $p$ while that of the non linear parts are $m$ and $k$. In our work, we considered a situation where the same 'orders' used in the linear parts are extended to the non linear component.

Besides, the above bilinear models have their orders begin with unity [that is, $l^{1}=1$ to $k$ in (2)] as lag values of $\varepsilon_{t}$ which could be misleading if we want to describe a pure bilinear AR process in the context of the linear form described below in equation (4). This stems from the fact that in linear AR process, only $X_{t}$ is lagged to give its 'order' description while the white noise remains in its present state and assumes lag zero. Again, we modified such concept by considering a case of zero lag values of white noise $\varepsilon_{t}$. Our interest also extended to estimation of the pure vector bilinear autoregressive (BIVAR) models. The data used for estimation is a three source monthly generated revenue (for a period of ten years) of Ikot Ekpene Local Government Area in Nigeria. Iwok and Etuk (2009) studied BIVARIMA models.

Theoretical framework and matrix formulation

The Linear Process (Univariate Case) : In its linear form, an AR of order $p$ denoted by AR (p) is given as

$X_{t}=\phi_{1} X_{t-1}+\phi_{2} X_{t-2}+\ldots+\phi_{p} X_{t-p}+\varepsilon_{t}$
Here $\varepsilon_{t}$ is in its present state and has lag zero.

As could be seen below, (4) forms part of the Autoregressive moving average (ARMA) process of order $(p, q)$ denoted by ARMA $(p, q)$ and expressed thus:

$$
X_{t}=\phi_{1} X_{t-1}+\ldots+\phi_{p} X_{t-p}+\varepsilon_{t}-\theta_{1} \varepsilon_{t-1}-\ldots-\theta_{q} \varepsilon_{t-q}
$$

The Mixed Bilinear Process (Univariate Case) :

The general bilinear model denoted by $B L(p, r, m, k)$ is expressed as:

$X_{t}=\sum_{i=1}^{p} a_{i} X_{t-i}+\sum_{j=1}^{q} c_{j} \varepsilon_{t-j}+\sum_{k=1}^{m} \sum_{j=1}^{k} b_{k j} X_{t-k} \varepsilon_{t-j}+\varepsilon_{t}$

where $\sum_{i=1}^{p} a_{i} X_{t-i}$ is the linear AR component with

order $\mathrm{p}, \sum_{j=1}^{q} c_{j} \varepsilon_{t-j}$ is the linear MA component with

order q, $\sum_{k=1}^{m} \sum_{j=1}^{k} b_{k j} X_{t-k} \varepsilon_{t-j}$ is the non linear part with

orders $(\mathrm{m}, \mathrm{k}),\left\{\varepsilon_{t}\right\}$ is the white noise.

Vector Autoregressive Bilinear Time Series: Since our matrix formulation will be based on pure AR process, we assume that the moving average (MA) component of the bilinear model vanishes by setting the MA parameters equal zero. But before the isolation process, we seek to define a vector of several time series as a multivariate case with respect to the 'orders' of their respective linear AR processes.

Firstly, let us consider an $n$-dimensional vector $\boldsymbol{X}_{\boldsymbol{t}}$ of time series:

$$
\boldsymbol{X}_{t}^{\prime}=\left[X_{1 t}, X_{2 t}, \ldots, X_{n t}\right] .
$$

Define $P$ to be a set of all AR orders of $X_{t}$; so that $p_{i} \in P$ and

$$
P=\left\{p_{1}, p_{2}, \ldots, p_{n}\right\} ; i=1,2, \ldots, n .
$$

While maintaining the AR orders, we distinguish the linear vector component matrix as:

$$
\boldsymbol{X}_{t}=\sum_{k=1}^{\max p_{i}} \gamma_{k} \boldsymbol{X}_{t-k}+U_{t}
$$

where $\gamma_{k}$ is an $n \times n$ matrix of linear component parameters.

By introducing a diagonal matrix $\boldsymbol{X}_{t-k}^{(D)}$ of lagged vector elements, the non linear part emerges: 
$\boldsymbol{X}_{\boldsymbol{t}}=\sum_{k=1}^{\max p_{i}} \boldsymbol{X}_{t-k}^{(D)}\left\{\beta_{k} \varepsilon_{t-l^{*}}\right\}+U_{t}$

$\beta_{k}$ is an $n \times n$ matrix of non linear component parameters.

Now combining the two equations above gives a bilinear vector autoregressive (BIVAR) matrix as shown:

$$
\boldsymbol{X}_{\boldsymbol{t}}=\sum_{k=1}^{\max p_{i}} \gamma_{k} \boldsymbol{X}_{t-k}+\sum_{k=1}^{\max p_{i}} \boldsymbol{X}_{t-k}^{(D)}\left\{\boldsymbol{\beta}_{k} \boldsymbol{\varepsilon}_{t-l^{*}}\right\}+U_{t}
$$

To isolate a pure BIVAR process, we set $l^{*}=0$

Thus:

$$
\boldsymbol{X}_{t}=\sum_{k=1}^{\max p_{i}} \gamma_{k} X_{t-k}+\sum_{k=1}^{\max p_{i}} \boldsymbol{X}_{t-k}^{(\boldsymbol{D})}\left\{\beta_{k} \varepsilon_{t-0}\right\}+\boldsymbol{U}_{t} .
$$$$
\text { where } U_{t} \text { is a white noise. }
$$

Also, to maintain the orders of the linear AR processes in our various bilinear expressions; the following conditions and definitions are applied in the matrix formulation:

i.) $\gamma_{k}=\left\{\gamma_{k, i j}\right\}_{n \times n}$ such that

$$
\gamma_{k . i j} \neq 0 \text { if } \exists X_{j t-k}
$$$$
\gamma_{k, i j}=0 \text { if } X_{j t-k} \text { does not exist. }
$$

ii. ) $\boldsymbol{\beta}_{k}=\left\{\beta_{k l^{*} . i j}\right\}_{n \times n}$
For pure BIVAR :

$$
\begin{aligned}
& \beta_{k}=\left\{\beta_{k 0 . i j}\right\}_{n \times n} \text { such that } \\
& \beta_{k 0 . i j} \neq 0 \text { if } \exists X_{i t-k} \\
& \beta_{k 0 . i j}=0 \text { if } X_{i t-k} \text { does not exist. }
\end{aligned}
$$

iii. ) $\boldsymbol{X}_{t-k}=\left\{X_{i t-k}\right\}_{n \times 1}$

iv. ) $\boldsymbol{X}_{t-k}^{(D)}=\operatorname{Diag}\left\{X_{i t-k}\right\}_{n \times n}$

v. ) $\boldsymbol{\varepsilon}_{t-0}=\left\{\varepsilon_{i t-0}\right\}_{n \times 1}$

$$
\text { Where } \varepsilon_{t-0} \text { is a white noise }
$$

process from $A R(p)$.

$$
\text { vi. ) } \boldsymbol{U}_{t}=\left\{u_{i t}\right\}_{n \times 1}
$$

$$
\boldsymbol{u}_{\boldsymbol{t}} \text { is an independent white }
$$

noise process

Hence we can write (10) as :

$\left.\left\{X_{i t}\right\}_{n \times 1}=\sum_{k=1}^{\max p_{i}}\left\{\gamma_{k . i j}\right\}_{n \times n}\left\{X_{i t-k}\right\}_{n \times 1}+\sum_{k=1}^{\max p_{i}}\left\{X_{i t-k}\right\}\right\}_{n \times n}^{(D)}\left[\left\{\beta_{k 0 . i j}\right\}_{n \times n}\left\{\varepsilon_{i t-0}\right\}_{n \times 1}\right]+\left\{u_{i t}\right\}_{n \times 1}$

We can simplify and expand the compressed matrices above as follows :

$$
\text { Let } p^{*}=\operatorname{maxp}_{i} \text {. }
$$

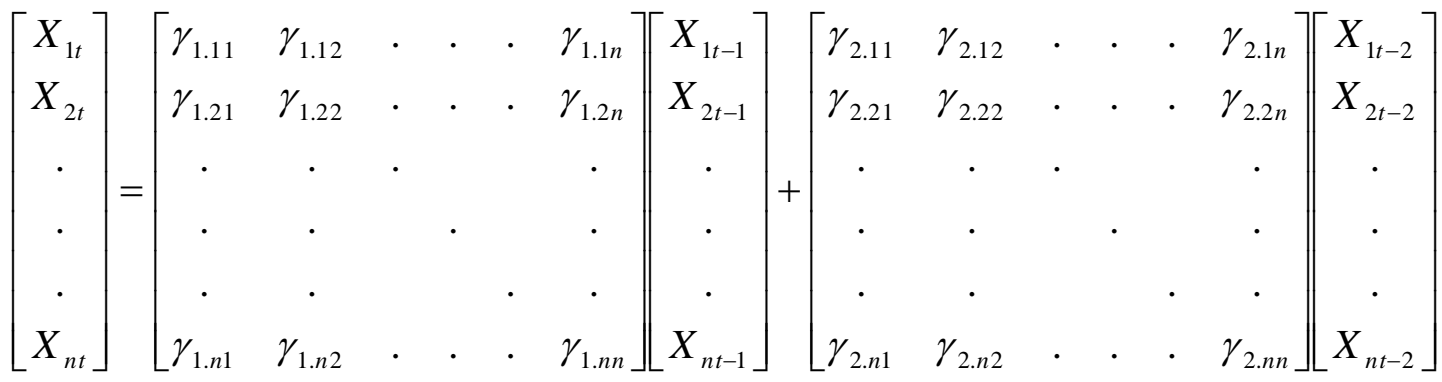

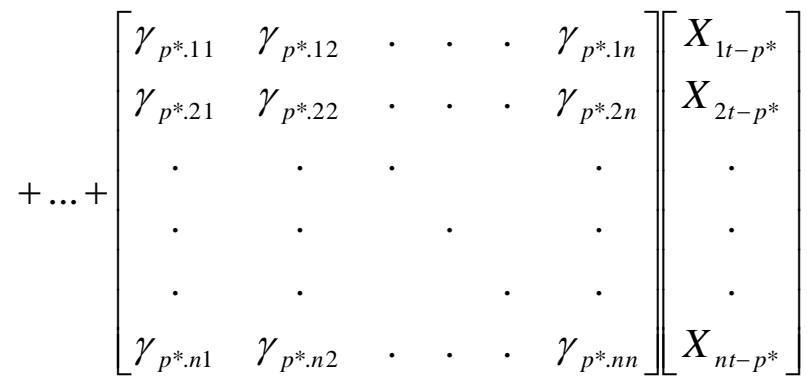




$$
\begin{aligned}
& +\left[\begin{array}{cccccc}
X_{1 t-1} & 0 & \cdot & \cdot & \cdot & 0 \\
0 & X_{2 t-1} & \cdot & \cdot & \cdot & 0 \\
\cdot & \cdot & \cdot & & \cdot \\
\cdot & \cdot & & \cdot & & \cdot \\
\cdot & \cdot & & & \cdot & \cdot \\
0 & 0 & \cdot & \cdot & \cdot & X_{n t-1}
\end{array}\right]\left\{\left[\begin{array}{cccccc}
\beta_{10.11} & \beta_{10.12} & \cdot & \cdot & \cdot & \beta_{10.1 n} \\
\beta_{10.21} & \beta_{10.22} & \cdot & \cdot & \cdot & \beta_{10.2 n} \\
\cdot & \cdot & \cdot & & \cdot \\
\cdot & \cdot & & \cdot & \cdot \\
\cdot & \cdot & & \cdot & \cdot \\
\beta_{10 . n 1} & \beta_{10 . n 2} & \cdot & \cdot & \cdot & \beta_{10 . n n}
\end{array}\right]\left[\begin{array}{c}
\varepsilon_{1 t-0} \\
\varepsilon_{2 t-0} \\
\cdot \\
\cdot \\
\cdot \\
\varepsilon_{n t-0}
\end{array}\right]\right\} \\
& +\left[\begin{array}{cccccc}
X_{1 t-2} & 0 & \cdot & \cdot & \cdot & 0 \\
0 & X_{2 t-2} & \cdot & \cdot & \cdot & 0 \\
\cdot & \cdot & \cdot & & & \cdot \\
\cdot & \cdot & & \cdot & & \cdot \\
\cdot & \cdot & & & \cdot & \cdot \\
0 & 0 & \cdot & \cdot & \cdot & X_{n t-2}
\end{array}\right]\left\{\left[\begin{array}{cccccc}
\beta_{20.11} & \beta_{20.12} & \cdot & \cdot & \cdot & \beta_{20.1 n} \\
\beta_{20.21} & \beta_{20.22} & \cdot & \cdot & \cdot & \beta_{20.2 n} \\
\cdot & \cdot & \cdot & & \cdot \\
\cdot & \cdot & \cdot & \cdot \\
\cdot & \cdot & & \cdot & \cdot \\
\beta_{20 . n 1} & \beta_{20 . n 2} & \cdot & \cdot & \cdot & \beta_{20 . n n}
\end{array}\right]\left[\begin{array}{c}
\varepsilon_{1 t-0} \\
\varepsilon_{2 t-0} \\
\cdot \\
\cdot \\
\cdot \\
\varepsilon_{n t-0}
\end{array}\right]\right\}
\end{aligned}
$$

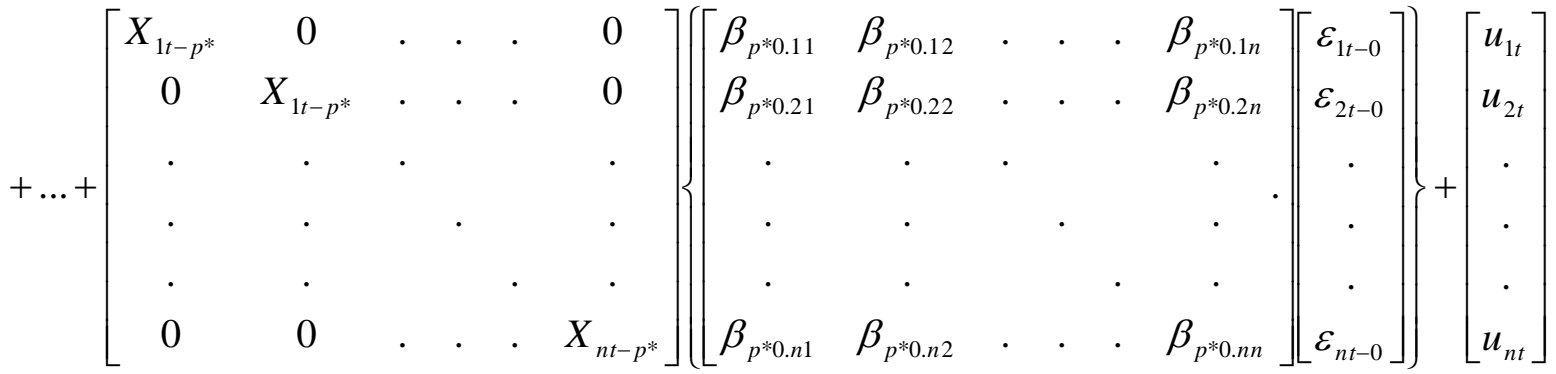

Recall that :

$$
\gamma_{k . i j} \neq 0 \text { iff } \exists X_{j t-k} \text { for the } k^{\text {th }}
$$

matrix ;

$$
\gamma_{k . i j}=0 \text {; otherwise. }
$$

Also: $\quad \beta_{k 0 . i j} \neq 0$ iff $\exists X_{i t-k}$ for the $k^{\text {th }}$ matrix ;

$$
\beta_{k 0 . i j}=0 \text {; otherwise. }
$$

If the above matrices are simplified further [that is multiplying, adding and equating both sides], we can conveniently establish a model for each vector element as:

$X_{i t}=\sum_{r=1}^{n} \sum_{k=1}^{\max p} \gamma_{k . i r} X_{r t-k}+\sum_{r=1}^{n} \sum_{k=1}^{\max p} \beta_{k 0 . i r} X_{i t-k} \varepsilon_{r t-0}+u_{t}$

where $\sum_{r=1}^{n} \sum_{k=1}^{\max p} \gamma_{k . i r} X_{r t-k}$ is the vector linear component;

$$
\sum_{r=1}^{n} \sum_{k=1}^{\max p} \beta_{k 0 . i r} X_{i t-k} \varepsilon_{r t-0} \text { is the vector non linear }
$$
component; $u_{t}$ is a white noise.

The above expression (12) though in vector form, isolate a pure Autoregressive bilinear model from mixed processes given by some authors in equations (1), (2) and (3). One interesting difference created by our models above is that the same AR orders used in the vector linear part are used in vector non linear part. These 'orders' are distributed accordingly in the linear and non linear components of the bilinear model. We now illustrate our point with the following example:

METHODS OF ESTIMATION AND ILLUSTRATION Let us consider a case of 3-dimensional vector of time series with reference to the data in appendix $A$ below. Here:

$$
\boldsymbol{X}_{t}^{\prime}=\left[X_{1 t}, X_{2 t}, X_{3 t}\right]
$$

The distribution of partial autocorrelation function of the non-stationary series suggests:

$$
X_{1 t} \text {, an AR(3) process, }
$$


$X_{2 t}$, an AR(2) process,

$X_{3 t}$, an $\mathrm{AR}(1)$ process.

Thus $P=\left\{p_{1}, p_{2}, p_{3}\right\}=\{3,2,1\}$

So that $\max . p=p^{*}=3$
Then the BIVAR matrix representation is given by :

$$
\boldsymbol{X}_{\boldsymbol{t}}=\sum_{k=1}^{3} \gamma_{k} \boldsymbol{X}_{\boldsymbol{t}-\boldsymbol{k}}+\sum_{k=1}^{3} \boldsymbol{X}_{\boldsymbol{t}-\boldsymbol{k}}^{(D)}\left\{\boldsymbol{\beta}_{k} \boldsymbol{\varepsilon}_{t-0}\right\}+\boldsymbol{U}_{\boldsymbol{t}}
$$

Expanding and simplifying the matrices, we have:

$$
\begin{aligned}
& {\left[\begin{array}{l}
X_{1 t} \\
X_{2 t} \\
X_{3 t}
\end{array}\right]=\left[\begin{array}{lll}
\gamma_{1.11} & \gamma_{1.12} & \gamma_{1.13} \\
\gamma_{1.21} & \gamma_{1.22} & \gamma_{1.23} \\
\gamma_{1.31} & \gamma_{1.32} & \gamma_{1.33}
\end{array}\right]\left[\begin{array}{l}
X_{1 t-1} \\
X_{2 t-1} \\
X_{3 t-1}
\end{array}\right]+\left[\begin{array}{lll}
\gamma_{2.11} & \gamma_{2.12} & 0 \\
\gamma_{2.21} & \gamma_{2.22} & 0 \\
\gamma_{2.31} & \gamma_{2.32} & 0
\end{array}\right]\left[\begin{array}{l}
X_{1 t-2} \\
X_{2 t-2} \\
X_{3 t-2}
\end{array}\right]+\left[\begin{array}{lll}
\gamma_{3.11} & 0 & 0 \\
\gamma_{3.21} & 0 & 0 \\
\gamma_{3.31} & 0 & 0
\end{array}\right]\left[\begin{array}{l}
X_{1 t-3} \\
X_{2 t-3} \\
X_{3 t-3}
\end{array}\right]} \\
& +\left[\begin{array}{ccc}
X_{1 t-1} & 0 & 0 \\
0 & X_{2 t-1} & 0 \\
0 & 0 & X_{3 t-1}
\end{array}\right]\left\{\left[\begin{array}{ccc}
\beta_{10.11} & \beta_{10.12} & \beta_{10.13} \\
\beta_{10.21} & \beta_{10.22} & \beta_{10.23} \\
\beta_{10.31} & \beta_{10.32} & \beta_{10.33}
\end{array}\right]\left[\begin{array}{c}
\varepsilon_{1 t-0} \\
\varepsilon_{2 t-0} \\
\varepsilon_{3 t-0}
\end{array}\right]\right\} \\
& +\left[\begin{array}{ccc}
X_{1 t-2} & 0 & 0 \\
0 & X_{2 t-2} & 0 \\
0 & 0 & X_{3 t-2}
\end{array}\right]\left\{\left[\begin{array}{ccc}
\beta_{20.11} & \beta_{20.12} & \beta_{20.13} \\
\beta_{20.21} & \beta_{20.22} & \beta_{20.23} \\
0 & 0 & 0
\end{array}\right]\left[\begin{array}{c}
\varepsilon_{1 t-0} \\
\varepsilon_{2 t-0} \\
\varepsilon_{3 t-0}
\end{array}\right]\right\} \\
& +\left[\begin{array}{ccc}
X_{1 t-3} & 0 & 0 \\
0 & X_{2 t-3} & 0 \\
0 & 0 & X_{3 t-3}
\end{array}\right]\left\{\left[\begin{array}{ccc}
\beta_{30.11} & \beta_{30.12} & \beta_{30.13} \\
0 & 0 & 0 \\
0 & 0 & 0
\end{array}\right]\left[\begin{array}{c}
\varepsilon_{1 t-0} \\
\varepsilon_{2 t-0} \\
\varepsilon_{3 t-0}
\end{array}\right]\right\}+\left[\begin{array}{l}
u_{1 t} \\
u_{2 t} \\
u_{3 t}
\end{array}\right]
\end{aligned}
$$

On simplifying the above matrices [that is multiplying, adding and equating both sides], we have for each vector element:

$$
X_{i t}=\sum_{r=1}^{3} \sum_{k=1}^{3} \gamma_{k . i r} X_{r t-k}+\sum_{r=1}^{3} \sum_{k=1}^{3} \beta_{k 0 . i r} X_{i t-k} \varepsilon_{r t-0}+u_{t}
$$

as expressed in equations (14), (15) and (16) below. To obtain the estimates of the parameters and fits, the resulting equation (13) is treated as an intrinsically linear model.
Estimates for the pure BIVAR models: The vector bilinear autoregressive process [see equations (12) and (13) ] consists of two parts. The first part is the linear vector AR process, while the second part is the product of lagged vector element and current state white noise. The distribution of autocorrelation and partial autocorrelation of the non-stationary series gives the order for each series of the vector; while our matrix formulation procedure distributes the lags. The regression parameter estimates obtained provide the following models for the three vector elements:

\section{RESULTS}

$$
\begin{aligned}
X_{1 t} & =0.266 X_{1 t-1}+0.0610 X_{2 t-1}+0.0322 X_{3 t-1}+0.397 X_{1 t-2}+0.163 X_{2 t-2}+0.178 X_{1 t-3} \\
& +0.00158 \varepsilon_{1 t-0} X_{1 t-1}-0.000724 \varepsilon_{2 t-0} X_{1 t-1}-0.000253 \varepsilon_{3 t-0} X_{1 t-1} \\
& +0.00196 \varepsilon_{1 t-0} X_{1 t-2}+0.000291 \varepsilon_{2 t-0} X_{1 t-2}-0.00132 \varepsilon_{3 t-0} X_{1 t-2} \\
& +0.000432 \varepsilon_{1 t-0} X_{1 t-3}+0.000918 \varepsilon_{2 t-0} X_{1 t-3}+0.00135 \varepsilon_{3 t-0} X_{1 t-3} \ldots \ldots \ldots \ldots \ldots . . .(14) \\
X_{2 t} & =0.0870 X_{1 t-1}+0.433 X_{2 t-1}-0.177 X_{3 t-1}+0.0903 X_{1 t-2}+0.497 X_{2 t-2}-0.0609 X_{1 t-3} \\
+ & 0.000036 \varepsilon_{1 t-0} X_{2 t-1}+0.00149 \varepsilon_{2 t-0} X_{2 t-1}+0.000549 \varepsilon_{3 t-0} X_{2 t-1} \\
+ & 0.000304 \varepsilon_{1 t-0} X_{2 t-2}+0.00260 \varepsilon_{2 t-0} X_{2 t-2}-0.00114 \varepsilon_{3 t-0} X_{2 t-2} \ldots \ldots \ldots \ldots \ldots . .(15)
\end{aligned}
$$


$X_{3 t}=0.0922 X_{1 t-1}-0.0544 X_{2 t-1}+0.651 X_{3 t-1}+0.0073 X_{1 t-2}+0.0079 X_{2 t-2}+0.0435 X_{1 t-3}$

$+0.000064 \varepsilon_{1 t-0} X_{3 t-1}-0.000374 \varepsilon_{2 t-0} X_{3 t-1}+0.0104 \varepsilon_{3 t-0} X_{3 t-1}$

could be seen above, these models are linear in states $X_{i t-k}$ but non-linear jointly with $\varepsilon_{i t-l}$ as the name 'bilinear' implies.

The zero lag white noise processes in the non linear parts clearly defines our models as pure BIVAR since it is only the vector elements that

are lagged. The estimates provided by the above models are displayed in Appendix $B$ and are found to be good, as evidenced by the closeness between the Actual and estimated values.
Plots of Actual and Estimate Values: The actual and estimated values of our models for each vector element presented in Appendix $A$ and $B$ are plotted in figures 1, 2 and 3 below. Each figure displayed contains two plots ( the actual marked by 'o' and the estimate marked by ' + ' ) As shown in the plots, there is a strong marriage between the actual and the estimates plot for each vector element and this indicates a good fit of our developed models.

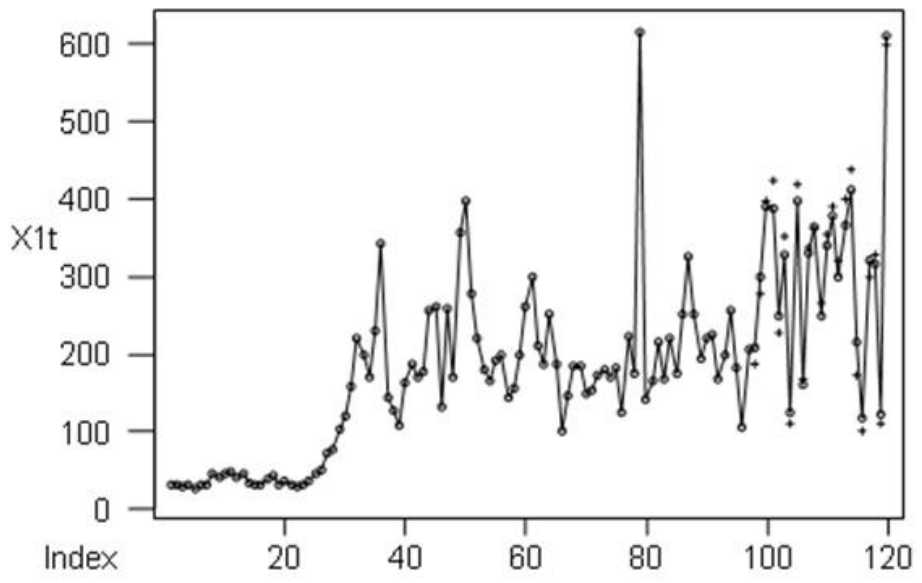

Key : o (actual plot) and + (estimates plot)

Fig 1 : Plots of actual and estimates of $X_{1 t}$ 
Am. J. Sci. Ind. Res., 2012, 3(2): 86-93

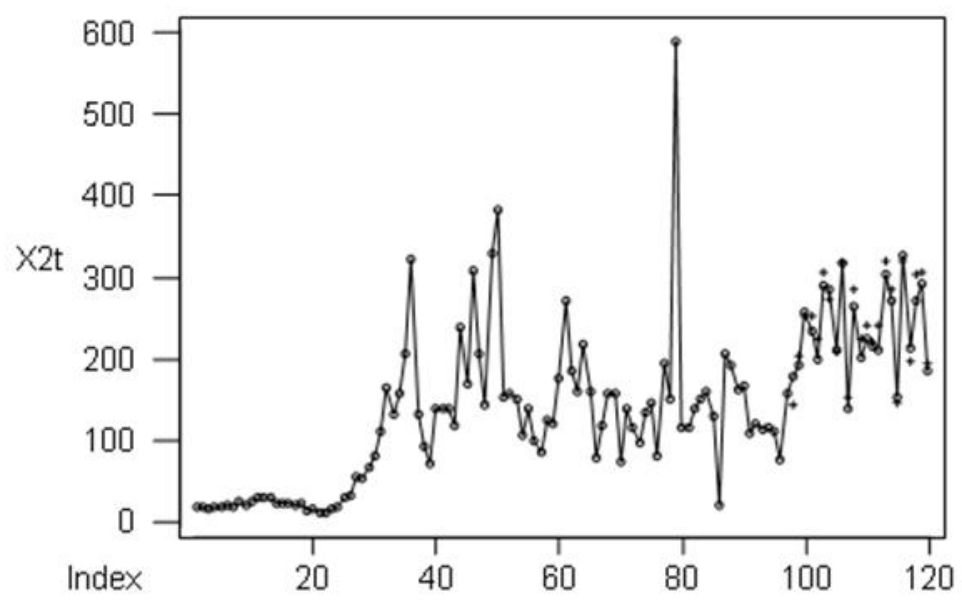

Key : o (actual plot) and + (estimates plot)

Fig 2 : Plots of actual and estimates of $X_{2 t}$

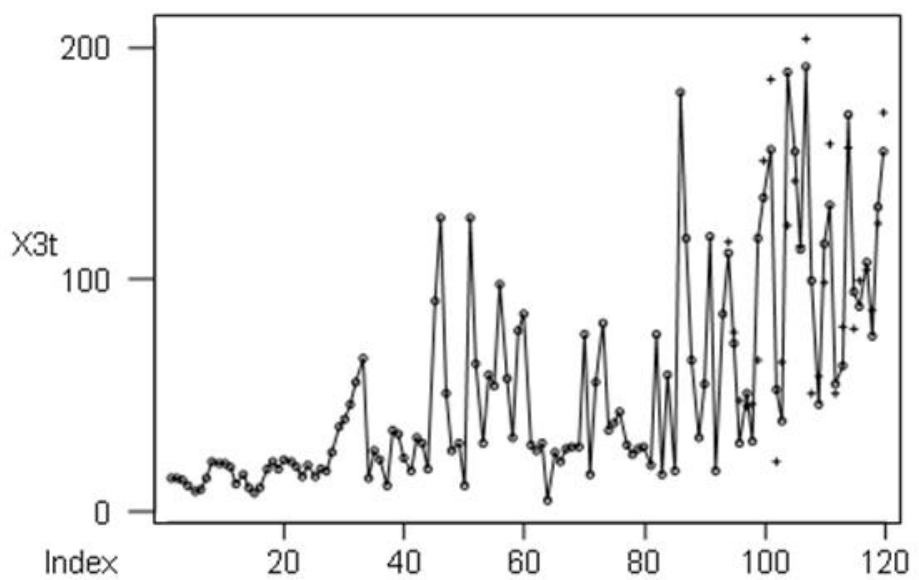

Key : o (actual plot) and + (estimates plot)

Fig 3 : Plots of actual and estimates of $X_{3 t}$. 


\section{SUMMARY AND CONCLUSIONS}

In essence, this work has established a bilinear concept from the conventional definition of a linear process in terms of 'order' by maintaining the present state of the white noise. The orders of the linear AR process obtained by Box and Jenkins method are used in both the linear and non linear parts of our derived bilinear models. These models are linear in states $X_{i t-k}$ and $\varepsilon_{i t-l}$ separately but non-linear jointly, as the name 'bilinear' implies. The parameter estimates of the generated models were used to obtain the estimate values of each vector element. The actual and estimate values plotted on each figure went together and in the same direction. This depicts the validity of our developed concepts. Hence there is no gain saying that our class of models offers another possibility of modeling non stationary series.

\section{DISCUSSION AND RECOMMENDATION}

Despite the beauty of such extension from linear to bilinear concept, the zero lagged white noise processes seem to restrict our progress in making forecasts. However, since our models comprise linear and non linear part; we suggest that forecasts be generated from the linear part and then used to forecast in our bilinear approach. The work is under progress to be treated in subsequent reports.

\section{REFERENCES}

Boonchai KS, Eivind S (2005). Multivariate bilinear time series; a stochastic alternative in population dynamics.Geophysical Research Abstracts, Vol.7,02219. SRef-ID:1607-7962/gra/EGU05-A02219.๑ European Geosciences Union 2005.http://www.cosis.net/abstract.

Bouzaachane K, Harti M, Benghabrit Y (2006). Parameter estimation for first-order superdiagonal bilinear time series. Interstat.statjournals.net/0607007.

Granger CWJ, Anderson AP (1978) Introduction to Bilinear Time Series Models. Vandenhoeck and Ruprecht, Göttingen, Germany, pp. $45-69$.

Iwok IA, Etuk EH (2009).On the Comparative Performance of Pure Vector Autoregressive-Moving Average and Vector Bilinear Autoregressive-Moving Average Time Series Models. Asian Journal Of Mathematics and Statistics, Vol. 2, No. 2, pp. $33-40$.

Maravall A (1983). An application of non linear time series forecasting. Journal of Business and Economic Statistics. 4: 66-74. http://www.tijsat.tu.ac.th/toc.id=2_diretory/vip.pdf.

Rao TS (1980).On the Theory of Bilinear Time Series Models. J.R. Statist. Soc. 43(2): 244-255.

Yule GU (1927). On a Method of Investigating the Periodicities in Disturbed Series, with special reference to Wolfer's Sunspot Numbers. Phil. Trans. Roy. Soc. London A: 226 - 267. 